\title{
Effects of supervised aerobic and strength training in overweight and grade I obese pregnant women on maternal and foetal health markers: the GESTAFIT randomized controlled trial
}

Virginia A. Aparicio ${ }^{1,2^{*}}$ (D), Olga Ocón ${ }^{3}$, Carmen Padilla-Vinuesa ${ }^{3,4}$, Alberto Soriano-Maldonado ${ }^{5,6}$, Lidia Romero-Gallardo ${ }^{6}$, Milkana Borges-Cósic ${ }^{6}$, Irene Coll-Risco ${ }^{1}$, Pilar Ruiz-Cabello ${ }^{1}$, Pedro Acosta-Manzano ${ }^{6}$, Fernando Estévez-López ${ }^{6,7}$, Inmaculada C. Álvarez-Gallardo ${ }^{6}$, Manuel Delgado-Fernández ${ }^{6}$, Jonatan R. Ruiz ${ }^{6}$, Mireille N. Van Poppel ${ }^{2,8+}$ and Julio J. Ochoa-Herrera ${ }^{1+}$

\begin{abstract}
Background: During pregnancy, a sedentary lifestyle may have negative consequences on maternal and foetal health status. The main objective of this project is to assess the effects of an exercise intervention in overweight and grade I obese pregnant on maternal and foetal health markers.

Methods/design: The present study aims to recruit 60 overweight and grade I obese women interested in participating in an exercise intervention program from the 17th gestational week until delivery. Women will be randomized to either an exercise (three 60-min sessions/week of combined aerobic and strength training and pelvic floor exercises), or usual care (control) group (30 women per group). The primary outcome measures are maternal weight gain, and maternal and neonatal glycaemic profile. Secondary outcome measures are: i) perinatal obstetric records; i) body composition; iii) dietary patterns; iv) physical fitness; v) low-back pain; vi) objectively measured physical activity and sedentary behaviour; vii) haematology and biochemical analyses; viii) oxidative stress; ix) pro- and anti-inflammatory markers; $x$ ) bone health biomarkers; xi) sleep quality; xii) mental health, quality of life and positive health.
\end{abstract}

Discussion: The findings of this project will help to identify strategies for primary prevention and health promotion based on this exercise-based intervention program among overweight and grade I obese pregnant women.

Trial registration: NCT02582567; Date of registration: 20/10/2015

Keywords: Physical activity, Physical fitness, Nutrition, Glycaemic profile, Oxidative stress, Quality of life, Newborn, Lipid profile, Sleep quality

\footnotetext{
*Correspondence: virginiaparicio@ugr.es

${ }^{\dagger}$ Equal contributors

'Department of Physiology, Faculty of Pharmacy and Institute of Nutrition and Food Technology, University of Granada, Granada, Spain

${ }^{2}$ Department of Public and Occupational Health, and EMGO+ Institute for

Health and Care Research, VU University medical center, Amsterdam, The

Netherlands

Full list of author information is available at the end of the article
} 


\section{Background}

Assessing the influence of lifestyle during pregnancy on maternal and foetal health is increasingly becoming a matter of interest [1-3]. Among these behaviours, physical activity (PA) may play an important role [4-8]. The sedentary lifestyle adopted by many pregnant women predisposes them to obesity, hypertension or gestational diabetes mellitus (GDM) [5, 6, 8], and the physiological changes occurring during gestation magnify this risk [9]. Importantly, overweight or obesity during pregnancy is associated with a significantly higher risk of pre-term delivery [10], birth-asphyxia-related complications [11], pre-eclampsia, GDM, prolonged labour, caesarean section, wound infection, postpartum haemorrhage, early neonatal death or neonatal intensive care admission [12-15], and infant mortality [16].

Maternal levels of PA may decline during pregnancy likely as a result of the physical changes of pregnancy and due to a combination of social and psychological factors, such as the thinking that resting during pregnancy is the safest behaviour $[17,18]$. However, increasing PA levels during pregnancy is effective in the prevention of GDM, hypertension, dysnea, excessive gestational weight gain, and high birth weight, among others $[5,7,8,19,20]$. Emerging evidence suggest that exercise training during pregnancy (including moderateto-high intensity exercise) might provide beneficial effects on both maternal and foetal health without side effects $[21,22]$. Indeed, it has been shown that exercise during gestation prevents diastasis recti abdominis [23] and that higher levels of strength or aerobic training are positively associated with hospital stay length, incidence of cesareans and Apgar test [24]. Further, strength training may reduce the need for insulin in overweight pregnant with GDM [25]. Finally, aerobic and strength exercise improve physical fitness and result in additional benefits [26, 27]. Moreover, during pregnancy, not only the DNA of a new life is created, also programmed through epigenetics. In this sense, maternal exercise may have benefits on the newborn, such as higher neurodevelopment [28, 29], better heart functioning, improved heart rate variability [30] and less body fat [28]. However, it is unknown the extent to which supervised exercise programs might improve inflammatory markers, antioxidant activity or bone biomarkers. Moreover, most of exercise programs conducted in pregnant women are performed at light-to-moderate exercise intensity, or are based on solely aerobic or strength training.

An optimal mental health during pregnancy is also a major concern. High levels of depression or anxiety during gestation and post-partum affect maternal quality of life, and in turn, could have a negative influence on the foetal and child health [31, 32]. Exercise may improve the pregnant quality of life and reduce stress [33-36], which might protect the foetus [31]. Also, adequate sleep during gestation improves quality of life [37], prevents stress and depression [38-40] and GDM [41, 42], reduces inflammatory signal [43] and the incidence of preeclampsia, premature birth or caesarea [44-46]. Further, optimal sleep in pregnancy is essential for the foetal development [47] and a better mother-child relationship [40]. However, pregnancy frequently affects sleep quality $[48,49]$ and it would of clinical relevance to better understand whether an exercise program positively influences the pregnant sleep quality.

Therefore, the main objective of the GESTAtion and FITness (GESTAFIT) project randomized controlled trial (RCT) is to assess the effects of a novel supervised exercise intervention developed in overweight and grade I obese pregnant on maternal and foetal health.

The present methodological article describes the study design, procedures and methods that will be conducted in the "GESTAFIT project".

\section{Methods/design Study design}

A supervised RCT (registration number: NCT02582567) based on an exercise program will be conducted in 60 overweight (body mass index $(\mathrm{BMI}) \geq 25-29.9)$, or grade I obese (BMI $\geq 30-34.9)$ pregnant women $(n=30$ in the exercise intervention group vs. 30 controls) from Granada (southeaster Spain). The organizational and participants flow is presented in Fig. 1.

The inclusion and exclusion criteria are described in Table 1. Participants will have to provide a written informed consent before taking part in the study, which will be conducted in accordance with the CONSORT (Consolidated Standards of Reporting Trials) statement [50]. Moreover, women will have to answer "No" to all questions from the PARmed-X for pregnancy health checklist (Physical Activity Readiness Medical Examination) by the Canadian Society for Exercise Physiology [51].

This study was approved by the Clinical Research Ethics Committee of Granada, Government of Andalusia, Spain (code: GESFIT-0448-N-15). The study will be conducted following the ethical guidelines of the Declaration of Helsinki, last modified in 2013.

\section{Recruitment process and measurements procedures}

The evaluation protocol scheme is shown in Table 2 . The measurements will be carried out in different days. The first assessment day will coincide with the 12th gestational week visit to the gynaecologist at "San Cecilio" University Hospital, Granada, and potential participants will be individually informed about the study objectives, evaluation protocol and procedures. If the woman agrees to participate in the present $\mathrm{RCT}$, the researcher will provide detailed information about each of the phases of 


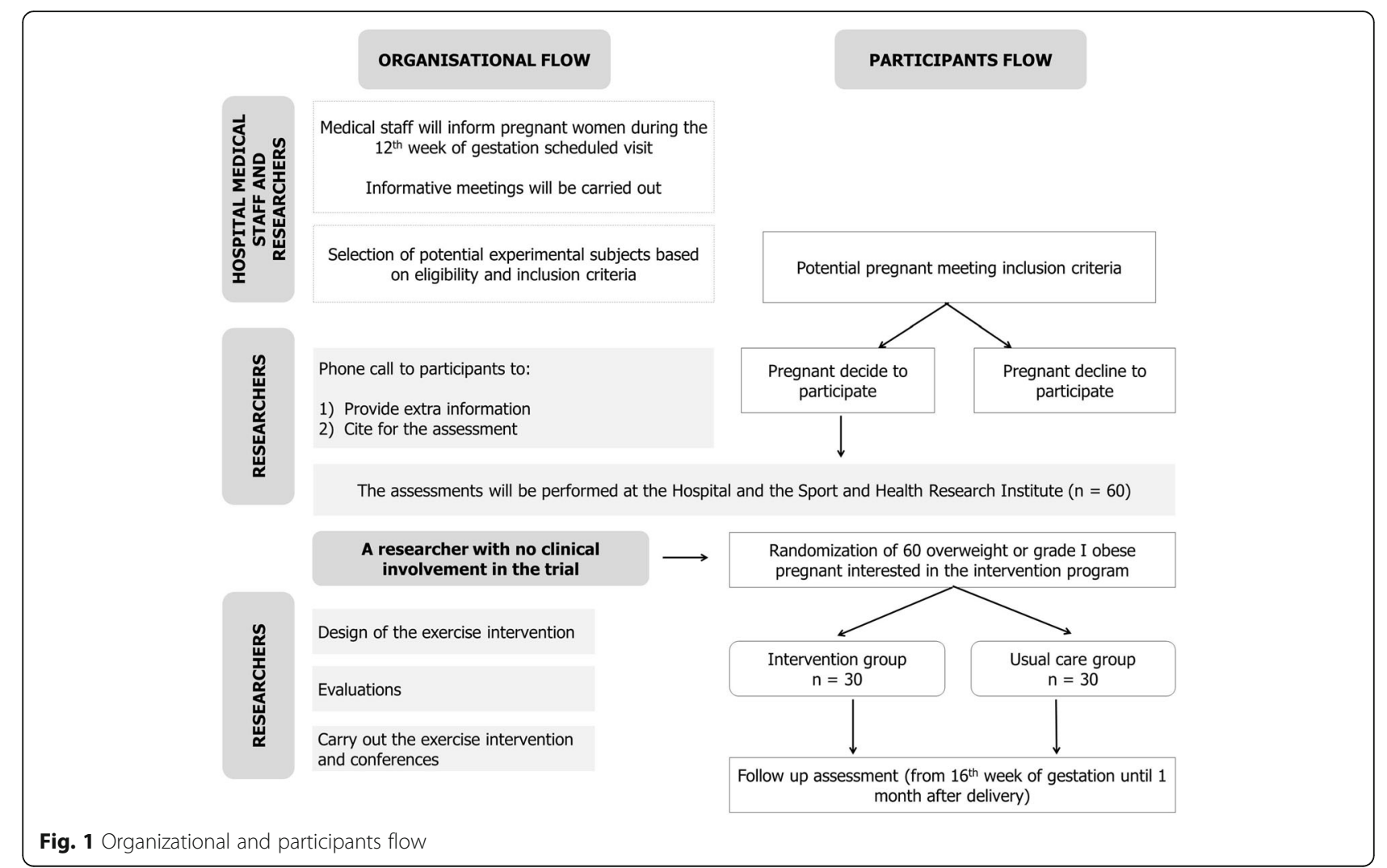

the study, and the participant will be asked to read and sign written informed consent. Subsequently (on the same day), weight and height will be assessed. On the second assessment day (16th gestational week) participants will attend the research centre and they will complete the following assessments: nutritional and clinical information, blood pressure, resting heart rate and physical fitness. The initial survey (anamnesis) will be conducted through face-to-face interviews by trained staff in order to gather data on sociodemographic characteristics, reproductive history, history of illness (hypertension, diabetes, obesity, etc.), and diet. Other health information will be collected using a self-administered questionnaire and it will include personal questions regarding smoking and alcohol habits and indicators of socio-demographic and socio-economic status (such as personal and household income, educational level or marital status). This survey will also include questions regarding the employment of nutritional supplements or special diets. Participant will then receive the accelerometer and questionnaires to be completed there or at home, and will be asked to return them eight days later. Additionally, the Hospital will make an appointment with the participants during that week for the biochemical and urine samples collection. The third day of evaluation will consist on accelerometry and sleep quality assessment on the 24th week of gestation. The fourth day of evaluation will take place on the 34th week of gestation. This evaluation will be exactly the same to that performed in the second day of evaluation before the exercise program started (i.e. 16th gestational week), including biochemical assessments.

Blood samples $(5 \mathrm{~mL})$ of all pregnant participants will be extracted at week 16th and 34th of gestation, and at delivery. Samples of venous and arterial blood from the umbilical cord $(5+5 \mathrm{~mL})$ at the time of delivery will be taken. In addition, samples of urine at 16 and 34 weeks gestation and delivery will be collected. All samples will be immediately frozen and conserved at $-80{ }^{\circ} \mathrm{C}$ to avoid breaking the cold chain before being sent to the laboratory.

\section{Sample size}

The number of participants to be included in the study was calculated on the basis of the change in body weight. We used the difference in weight change observed between the control and intervention group (1.04 $\mathrm{Kg}$ ) in Ruiz et al. [20] as the expected effect size. A total of 52 women ( 26 per group) will be needed to detect a mean group difference of 1.04 and a standard deviation of $1.15 \mathrm{Kg}$ in weight change with a power of $90 \%$ and $\alpha$ of 0.05 . However, we will exceed this sample size to allow for withdrawals. Assuming a maximum lost-to- 
Table 1 Inclusion and exclusion criteria in the GESTAFIT project

Inclusion criteria
- Overweight or grade I obese pregnant woman aged 25-40 years
old with a normal pregnancy course.
- Answer "no" to all questions on the PARmed-X for pregnancy*.
- To be able to walk without assistance.
- To be able to read and write enough.
- Informed consent: To be capable and willing to provide consent.
*In addition, specific inclusion criteria for data analysis are: gestational
age at delivery of $37-42$ weeks with single foetus, spontaneous
vaginal delivery or instrumental vaginal and caesarean without
maternofoetal pathology (or other indication that does not involve
maternofoetal risk, such as disproportion, failed induction, no foetal
progression or non-cephalic presentation), newborn with appropriate
weight, Apgar score > 7 in the 1st and 5 th minute of life, cord blood
pH (normal > 7.20) and normal monitoring results.
Exclusion criteria
- Acute or terminal illness.
- Malnutrition.
- Inability to conduct tests for assessing physical fitness or exercise
during pregnancy.
- Underweight, normal-weight or grade II-III obesity.
- Pregnancy risk factors (such as hypertension, type 2 diabetes, etc.).
- Multiple pregnancies.
- Chromosopathy or foetal malformations.
- Unterine growth restriction.
- Foetal death.
- Upper or lower extremity fracture in the past 3 months.
- Presence of neuromuscular disease or drugs affecting
- Be registered in other exercise program
- Perform more than 300 min of at least moderate physical activity
per for pregnancy: Physical Activity Readiness Medical Examination
-

PARmed-X for pregnancy: Physical Activity Readiness Medical Examination

follow up of $15 \%$ [20], we will recruit a total of 30 participants per group $(n=60)$.

\section{Randomization and blinding}

After all the baseline evaluations, participants will be randomized to either the exercise intervention or the control group. A computer generated simple randomization sequence will be created before participants will be enrolled, to allocate participants to either group (1:1). The randomization sequence will be prepared by a member of the research team with no clinical involvement in the trial. The allocation will be concealed in a password protected computer file. Whereas the participants will be aware of their group allocation, outcome assessors and data analysts will be blinded to the allocation.

\section{Exercise intervention}

Most of exercise programs developed for pregnant women until now [20, 27, 52] have been designed in compliance with the recommendations of the American College of Obstetrics and Gynaecology (ACOG) in 2002 [53], or the Canadian Society for Exercise Physiology, among others [54]. However, in 2011, Zavorsky \& Longo [55] launched a more actual, specific, and evidencebased guideline for exercise programs during pregnancy. Consequently, the exercise program that will be conducted in the present project has been developed accordingly to this guideline [55]. Nonetheless, we have also followed similar studies $[56,57]$ where aerobic and resistance training, as well as strengthening of the pelvic floor muscles [58], have been developed successfully and in line with these recommendations [57].

The exercise intervention $(n=30)$ will be performed in three groups of 10 participants and will meet the training standards of the American College of Sports Medicine [59] for adults. The groups will train 3 days/week (55-60 minutes per session) from the 17th week of gestation until delivery. The exercise sessions will be designed, carefully supervised, guided and instructed by qualified exercise professionals with experience in working with pregnant women.

The exercise program planning is shown in Table 3. The exercise intervention group will have different phases: a) Information phase: which will involve the understanding of the intervention plan for them, the goals we want to work with them, and how we will perform it (one training session); b) Movement Learning phase: theoretical and practical sessions with the explanation of movements and explanation of basic movement patterns (two training sessions); and c) Physical Fitness Training phase: with training sessions aimed at improving fitness and weight management (from the 18th until the 34th gestational week), and training sessions focused on a correct pelvic mobilization for the delivery (after the 34th gestational week until delivery) in order to try to reduce caesarean section rate, delivery time and number of pushes [23, 60].

The exercise intensity will be prescribed as percentages of heart rate reserve (\% HRR) [55] and the ratings of perceived exertion (RPE) using the Borg 6-20 RPE scale [61]. We will also employ the Karvonen formula to estimate the training heart rate (e.g. $65 \%$ HRR for a pregnant with $185 \mathrm{bpm}$ maximum heart rate and $80 \mathrm{bpm}$ basal heart rate would be calculated as: Training Heart Rate $=0.65 *(185-80)+80)$. Heart rate will be measured with heart rate monitors (Polar Electro OY, Finland) to control the intensity of the sessions. One third of the participants in the intervention group will wear heart rate monitors in $1 / 3$ of the sessions, both randomly selected. Intensity (expressed as RPE) will be expected to range from 12 to 16 . 
Table 2 Evaluation protocol scheme

\begin{tabular}{|c|c|c|c|c|c|c|}
\hline & $\begin{array}{l}\text { Criteria } \\
\text { (12th week) }\end{array}$ & $\begin{array}{l}\text { Baseline } \\
\text { (16th week) }\end{array}$ & $\begin{array}{l}\text { Second trimester } \\
\text { (24th week) }\end{array}$ & $\begin{array}{l}\text { Third trimester } \\
\text { (34th week) }\end{array}$ & Delivery & $\begin{array}{l}1 \text { month } \\
\text { post-partum }\end{array}$ \\
\hline Informed consent & $x$ & & & & & \\
\hline \multicolumn{7}{|l|}{ 1st day evaluation } \\
\hline Sociodemographic data & & $x$ & & & & \\
\hline Clinical history & & $x$ & & $x$ & $x$ & $x$ \\
\hline Weight and height & $x$ & $x$ & & $x$ & & $x$ \\
\hline $\begin{array}{l}\text { Body composition (dual-energy x-ray } \\
\text { absorptiometry) }\end{array}$ & & & & & & $x$ \\
\hline Accelerometry & & $x$ & $x$ & $x$ & & \\
\hline Physical fitness & & $x$ & & $x$ & & \\
\hline Nutritional study & & $x$ & & $x$ & & $x$ \\
\hline \multicolumn{7}{|c|}{ Questionnaires to be fill there or at home between the 1st and 2nd evaluation days } \\
\hline Low back-pain & & $x$ & & $x$ & & $x$ \\
\hline Quality of life & & $x$ & & $x$ & & $x$ \\
\hline Sleep quality & & $x$ & $x$ & $x$ & & $x$ \\
\hline Physical activity & & $x$ & $x$ & $x$ & & $x$ \\
\hline Self-perceived physical fitness & & $x$ & $x$ & $x$ & & $x$ \\
\hline Sexual function & & $x$ & & $x$ & & $x$ \\
\hline Restless legs syndrome & & $x$ & & $x$ & & $x$ \\
\hline Mental and positive health & & $x$ & & $x$ & & $x$ \\
\hline $\begin{array}{l}\text { 2nd day evaluation: Accelerometers collection and } \\
\text { review of questionnaires }\end{array}$ & & $x$ & & $x$ & & \\
\hline Maternal biochemical analysis & & $x$ & & $x$ & $x$ & \\
\hline Umbilical cord blood sampling & & & & & $x$ & \\
\hline Maternal urine sampling & & $x$ & & $x$ & $x$ & \\
\hline
\end{tabular}

The intensity will be adapted during the exercise program based on the week of gestation and each woman's heart rate. The Physical Fitness Training phase sessions until the 34th gestational week will consist of mixed work, composed of circuits where both muscular and cardiovascular conditioning will be implemented. This type of exercise training has been already developed by White et al. [57] who demonstrated better results for both the prevention of GDM, preeclampsia and preterm births, and for increased vaginal vs. caesarean deliveries.

Each training session will include $10 \mathrm{~min}$ warm-up with walks, mobility and activation exercises. The main part will consist of 40 min exercises organized in circuit. The circuit will alternate muscular and cardiovascular blocks of concurrent training. Each muscle circuit will consist of a hip dominant movement exercise (e.g. deadlift exercises, hinge hip, swing hip), a dominant knee (e.g. squats, lunges) 2 pull movements, 1 push movement (push-ups adapted) and 1 CORE muscles movement. Cardiovascular blocks will take approximately $3 \mathrm{~min}$ and will be composed of aerobic exercises as different variants of step-ups, small choreographies, front and side trips. Sessions will finish with a $10 \mathrm{~min}$ cool- down period of stretching, breathing, relaxation exercises and myofascial relief [62]. None of the proposed exercises will include Valsalva manoeuvre, supine positions, or high impact exercises that could go in detriment of the successful activation of the lumboabdominal belt, promote a decrease in venous return or alterations in blood pressure, among others. The exercise program volume and intensity programmed is also shown in Table 3.

To maximize adherence, several strategies will be implemented including music in all sessions and telephone calls following missed sessions. The researchers will control and register the presence of adverse events during the class and between classes.

The participants randomly assigned to the control group $(n=30)$ will receive general advices about the positive effects of PA during pregnancy for her and the foetus's health status. The researchers will give three seminar explaining 1) the benefits of exercise for a better pregnancy, prevention and treatment of GDM and excessive weight gain; 2) ergonomic advises, exercises to perform at home (e.g. stretching, resistance training) and strategies to increase their daily PA levels; 3 ) the 
Table 3 Supervised exercise intervention program

\begin{tabular}{|c|c|c|c|c|c|c|c|c|c|c|c|}
\hline $\begin{array}{l}\text { SESION } \\
\text { STRUCTURE }\end{array}$ & \multicolumn{11}{|l|}{ CONTENT } \\
\hline $\begin{array}{l}\text { WARM-UP } \\
10 \mathrm{~min}\end{array}$ & \multicolumn{11}{|c|}{ Joint mobility and different walk modalities } \\
\hline \multirow{18}{*}{$\begin{array}{l}\text { CONDITIONING } \\
40 \mathrm{~min}\end{array}$} & Training week & 1 & $2-4$ & $5-6$ & $7-8$ & $9-10$ & $11-12$ & $13-14$ & $15-16$ & $17-18$ & $>19$ \\
\hline & Gestational week & 17 & $18-20$ & $21-22$ & $23-24$ & $25-26$ & $27-28$ & $29-30$ & $31-32$ & $33-34$ & $>34$ \\
\hline & Intensity (RPE) & & $12-13$ & $12-13$ & $13-14$ & $13-14$ & $14-15$ & $14-15$ & $15-16$ & $15-16$ & \\
\hline & \multirow{7}{*}{$\begin{array}{l}\text { Monday } \\
\text { CIRCUIT (muscular and } \\
\text { cardiovascular blocks) }\end{array}$} & \multirow{7}{*}{$\begin{array}{l}\text { Familiarization and } \\
\text { acquisition of the basic } \\
\text { movement patterns }\end{array}$} & $5 \mathrm{RE} \times 3$ & $5 \mathrm{RE} \times 3$ & $5 \mathrm{RE} \times 3$ & $5 \mathrm{RE} \times 3$ & $5 \mathrm{RE} \times 3$ & $5 \mathrm{RE} \times 3$ & $5 \mathrm{RE} \times 3$ & $5 \mathrm{RE} \times 3$ & \multirow{7}{*}{$\begin{array}{l}\text { Pelvic movements + integration } \\
\text { pattern. Real transfer to delivery } \\
\text { moment }\end{array}$} \\
\hline & & & $\begin{array}{l}1 \text { min } \\
\text { REST }\end{array}$ & $\begin{array}{l}1 \text { min } \\
\text { REST }\end{array}$ & $\begin{array}{l}1 \text { min } \\
\text { REST }\end{array}$ & $\begin{array}{l}1 \text { min } \\
\text { REST }\end{array}$ & $\begin{array}{l}1 \text { min } \\
\text { REST }\end{array}$ & $\begin{array}{l}1 \text { min } \\
\text { REST }\end{array}$ & $\begin{array}{l}1 \text { min } \\
\text { REST }\end{array}$ & $\begin{array}{l}1 \text { min } \\
\text { REST }\end{array}$ & \\
\hline & & & $1 \mathrm{AE} 5^{\prime}$ & $1 \mathrm{AE} 5^{\prime}$ & $1 \mathrm{AE} 5^{\prime}$ & 1 AE $5^{\prime}$ & $1 \mathrm{AE} 5^{\prime}$ & $1 \mathrm{AE} 5^{\prime}$ & $1 \mathrm{AE} 5^{\prime}$ & $1 \mathrm{AE} 5^{\prime}$ & \\
\hline & & & $\begin{array}{l}1 \text { min } \\
\text { REST }\end{array}$ & $\begin{array}{l}1 \text { min } \\
\text { REST }\end{array}$ & $\begin{array}{l}1 \text { min } \\
\text { REST }\end{array}$ & $\begin{array}{l}1 \text { min } \\
\text { REST }\end{array}$ & $\begin{array}{l}1 \text { min } \\
\text { REST }\end{array}$ & $\begin{array}{l}1 \text { min } \\
\text { REST }\end{array}$ & $\begin{array}{l}1 \text { min } \\
\text { REST }\end{array}$ & $\begin{array}{l}1 \mathrm{~min} \\
\text { REST }\end{array}$ & \\
\hline & & & $5 R E \times 3$ & $5 R E \times 3$ & $5 R E \times 3$ & $5 R E \times 3$ & $5 R E \times 3$ & $5 R E \times 3$ & $5 R E \times 3$ & $5 R E \times 3$ & \\
\hline & & & 1AE 5' & $1 \mathrm{AE} 5^{\prime}$ & $1 \mathrm{AE} 5^{\prime}$ & $1 \mathrm{AE} 5^{\prime}$ & $1 \mathrm{AE} 5^{\prime}$ & $1 \mathrm{AE} 5^{\prime}$ & $1 \mathrm{AE} 5^{\prime}$ & $1 \mathrm{AE} 5^{\prime}$ & \\
\hline & & & $\begin{array}{l}1 \text { min } \\
\text { REST }\end{array}$ & $\begin{array}{l}1 \text { min } \\
\text { REST }\end{array}$ & $\begin{array}{l}1 \text { min } \\
\text { REST }\end{array}$ & $\begin{array}{l}1 \text { min } \\
\text { REST }\end{array}$ & $\begin{array}{l}1 \text { min } \\
\text { REST }\end{array}$ & $\begin{array}{l}1 \text { min } \\
\text { REST }\end{array}$ & $\begin{array}{l}1 \text { min } \\
\text { REST }\end{array}$ & $\begin{array}{l}1 \text { min } \\
\text { REST }\end{array}$ & \\
\hline & $\begin{array}{l}\text { Wednesday } \\
\text { (cardiovascular block) }\end{array}$ & $\begin{array}{l}\text { Familiarization and } \\
\text { acquisition of the basic } \\
\text { movement patterns }\end{array}$ & \multicolumn{8}{|c|}{ Choreographies and aerobic exercises } & $\begin{array}{l}\text { Pelvic movements + integration } \\
\text { pattern. Real transfer to delivery } \\
\text { moment }\end{array}$ \\
\hline & \multirow{7}{*}{$\begin{array}{l}\text { Friday } \\
\text { CIRCUIT (muscular and } \\
\text { cardiovascular blocks) }\end{array}$} & \multirow{7}{*}{$\begin{array}{l}\text { Familiarization and } \\
\text { acquisition of the basic } \\
\text { movement patterns }\end{array}$} & $5 \mathrm{RE} \times 3$ & $5 R E \times 3$ & $5 \mathrm{RE} \times 3$ & $5 \mathrm{RE} \times 3$ & $5 \mathrm{RE} \times 3$ & $5 \mathrm{RE} \times 3$ & $5 \mathrm{RE} \times 3$ & $5 \mathrm{RE} \times 3$ & \multirow{7}{*}{$\begin{array}{l}\text { Pelvic movements + integration } \\
\text { pattern. Real transfer to delivery } \\
\text { moment }\end{array}$} \\
\hline & & & $\begin{array}{l}1 \text { min } \\
\text { REST }\end{array}$ & $\begin{array}{l}1 \text { min } \\
\text { REST }\end{array}$ & $\begin{array}{l}1 \text { min } \\
\text { REST }\end{array}$ & $\begin{array}{l}1 \text { min } \\
\text { REST }\end{array}$ & $\begin{array}{l}1 \text { min } \\
\text { REST }\end{array}$ & $\begin{array}{l}1 \text { min } \\
\text { REST }\end{array}$ & $\begin{array}{l}1 \text { min } \\
\text { REST }\end{array}$ & $\begin{array}{l}1 \text { min } \\
\text { REST }\end{array}$ & \\
\hline & & & $1 \mathrm{AE} 5^{\prime}$ & $1 \mathrm{AE} 5^{\prime}$ & $1 \mathrm{AE} 5^{\prime}$ & 1 AE $5^{\prime}$ & $1 \mathrm{AE} 5^{\prime}$ & $1 \mathrm{AE} 5^{\prime}$ & $1 \mathrm{AE} 5^{\prime}$ & $1 \mathrm{AE} 5^{\prime}$ & \\
\hline & & & $\begin{array}{l}1 \text { min } \\
\text { REST }\end{array}$ & $\begin{array}{l}1 \text { min } \\
\text { REST }\end{array}$ & $\begin{array}{l}1 \text { min } \\
\text { REST }\end{array}$ & $\begin{array}{l}1 \text { min } \\
\text { REST }\end{array}$ & $\begin{array}{l}1 \text { min } \\
\text { REST }\end{array}$ & $\begin{array}{l}1 \text { min } \\
\text { REST }\end{array}$ & $\begin{array}{l}1 \text { min } \\
\text { REST }\end{array}$ & $\begin{array}{l}1 \text { min } \\
\text { REST }\end{array}$ & \\
\hline & & & $5 R E \times 3$ & $5 R E \times 3$ & $5 R E \times 3$ & $5 R E \times 3$ & $5 R E \times 3$ & $5 R E \times 3$ & $5 R E \times 3$ & $5 R E \times 3$ & \\
\hline & & & $1 \mathrm{AE} 5^{\prime}$ & $1 \mathrm{AE} 5^{\prime}$ & $1 \mathrm{AE} 5^{\prime}$ & $1 \mathrm{AE} 5^{\prime}$ & $1 \mathrm{AE} 5^{\prime}$ & $1 \mathrm{AE} 5^{\prime}$ & $1 \mathrm{AE} 5^{\prime}$ & $1 \mathrm{AE} 5^{\prime}$ & \\
\hline & & & $\begin{array}{l}1 \text { min } \\
\text { REST }\end{array}$ & $\begin{array}{l}1 \mathrm{~min} \\
\text { REST }\end{array}$ & $\begin{array}{l}1 \mathrm{~min} \\
\text { REST }\end{array}$ & $\begin{array}{l}1 \mathrm{~min} \\
\text { REST }\end{array}$ & $\begin{array}{l}1 \mathrm{~min} \\
\text { REST }\end{array}$ & $\begin{array}{l}1 \mathrm{~min} \\
\text { REST }\end{array}$ & $\begin{array}{l}1 \mathrm{~min} \\
\text { REST }\end{array}$ & $\begin{array}{l}1 \mathrm{~min} \\
\text { REST }\end{array}$ & \\
\hline $\begin{array}{l}\text { COOL-DOWN } \\
10 \mathrm{~min}\end{array}$ & \multicolumn{11}{|c|}{ Myofascial release, stretching and relaxation exercises } \\
\hline
\end{tabular}

RPE rating of perceived exertion, $R E$ resistance exercise, $A E$ aerobic exercise, REST resting. The load will be gradually and individualized increased for each participant to reach the intensity designed for each session 
benefits of the Mediterranean Diet and nutritional education. Moreover, we will prepare brochures describing the overall benefits of PA on health, and we will dispense general guidelines to increase the level of daily PA and optimum nutrition during pregnancy [63, 64].

\section{Primary and secondary outcomes \\ Primary outcome}

The primary outcomes are maternal weight gain and maternal and foetal glycaemic profile. Maternal weight gain will be defined as the weight change from baseline measurement to the last measurement at 34th week of gestation. Maternal insulin sensitivity will be derived from the homeostatic model assessment for insulin resistance (HOMA-IR), which will be calculated using the formula [fasting insulin $(\mu \mathrm{IU} / \mathrm{mL}) \times$ fasting glucose $(\mathrm{mg} / \mathrm{dL})] / 405$ in the 34th week of gestation. Neonatal insulin sensitivity will be assessed through the cord blood ratio glucose/insulin.

\section{Secondary outcomes}

\section{Maternal and foetal outcomes}

Birth weight; gestational age; type of delivery (natural, instrumental, or caesarean); Apgar scores (at 1 and $5 \mathrm{~min}$ ); time of dilation, expulsion, and childbirth; GDM; and hypertension, will be obtained from perinatal obstetric records.

\section{Blood pressure and resting heart rate}

Systolic and diastolic blood pressure, as well as resting heart rate, will be measured after 5 min of rest, on 2 separate occasions (with 2 min between trials), with the person seated (Omron Health Care Europe B.V. Hoolddorp). The lowest value of the two trials will be selected for the analysis.

\section{Maternal and foetal anthropometry and body composition}

Maternal Height will be measured only at the baseline measurement with a stadiometer (Seca 22, Hamburg). Pre-pregnancy weight will be based on self-report. Height measurement and pre-pregnancy weight will be used to calculate BMI (weight[Kg]/height[m2]) [65].

One month after delivery (postpartum period) lean, fat and bone mass of the whole body will be measured using a dual-energy $\mathrm{x}$-ray absorptiometry (DXA) device (Hologic Discovery QDR, Nasdaq: HOLX). Waist circumference $(\mathrm{cm})$ will be assessed at the middle point between the ribs and the ileac crest, with the participant standing (Harpenden anthropometric tape, Holtain Ltd).

Foetus/newborn A monitoring of the evolution of the foetal anthropometrics will be performed by obstetrical ultrasound measurements following the standards of the International Society of Ultrasound in Obstetrics and
Gynaecology guidelines and the methodology by Perin et al. [66]. The baby also will have a physical examination within $24 \mathrm{~h}$ after birth. Baby's weight, length and head circumference will be measured. Newborns will be classified as having low birth weight or macrosomia when birth weight was less than $2500 \mathrm{~g}$ and greater than $4000 \mathrm{~g}$, respectively.

\section{Dietary patterns}

The Mediterranean Diet Score [67] in a version adapted to the specific needs during pregnancy for $\mathrm{Fe}, \mathrm{Ca}$ and folic acid [68] will be used to assess the adherence to the traditional Mediterranean dietary pattern. A moderate alcohol diet, also typical of the Mediterranean Diet, will not be considered for calculating the index in this group of women, who should not drink alcohol due to their pregnancy.

The frequency of consumption of foods will be also studied by means of a quantitative questionnaire, validated and designed by Mataix et al. [69].

\section{Physical fitness}

Participants' physical fitness status in the 16th and 34th week of pregnancy will be assessed by means of the following tests:

Cardiorespiratory fitness Aerobic capacity will be estimated with a submaximal cycle ergometer heart rate method. A pedaling rate of $50 \mathrm{rpm}$ will kept constant by use of a metronome. Initial workload, $50 \mathrm{~W}$ or $75 \mathrm{~W}$, will be based on the women's reported physical activity levels, and increased by $25 \mathrm{~W}$ per minute until a steady state heart rate of 125 - beats or more per minute was reached, after which the women will cycle for at least 6 min until two consecutive heart rates, one minute apart, differ by 3 or fewer beats per minute. The $\mathrm{VO}_{2 \max }$ will be calculated according to the Åstrand and Ryhming nomogram [70].

We will additionally perform the 6-min walk test, which measures the maximum distance (in meters) each participant can walk in 6 min along a $45.7 \mathrm{~m}$ rectangular course [71].

Upper-body muscle strength The handgrip strength will be measured using a digital dynamometer (TKK 5101 Grip-D; Takey, Tokyo, Japan) as described elsewhere [72]. The participants will perform (alternately with both hands) these tests twice. The best value of 2 trials for each hand will be chosen and the average of both hands will be used in the analyses.

Upper-body flexibility The back-scratch test, a measure of overall shoulder range of motion, involves measuring the distance between (or overlap of) the middle fingers 
behind the back with a ruler [71]. The best score of two attempts for each arm in centimetres will be recorded and the average of both arms will be used in the analyses.

Self-perceived physical fitness The International Fitness Scale [73] is composed of five Likert-scale questions asking about the perceived participants' overall fitness, cardio-respiratory fitness, muscular fitness, speed-agility and flexibility in comparison with their friends' physical fitness ("very poor", "poor", "average", "good" and "very good").

\section{Low-back pain}

Pregnant women usually report low-back pain, especially in the third gestational trimester. Low-back pain will be assessed with the Spanish version of the Oswestry Disability Index score [74]. We will additionally employ the Pain Visual Analogue Scale to assess low back pain intensity [75].

\section{Physical activity and sedentary behaviour}

Accelerometry will be used to objectively assess PA and sedentary time. Women will be asked to wear a tri-axial accelerometer (ActiSleep+, Pensacola, Florida, United States) for 9 consecutive days, starting the same day they receive the monitor (e.g. participants who receive the accelerometer on Monday, will carry the device until Tuesday of the next week). The first and last day will be excluded from the analyses, accounting for a total of 7 days of registering. Participants will be instructed to wear the accelerometer during the whole day $(24 \mathrm{~h})$ on their wrist attached by an elastic belt. To prevent any damage to the devices, these will be taken off during water-based activities such as bathing or swimming. Time engaged in light, moderate, and moderate-vigorous intensity PA and sedentary time will be calculated. This accelerometer has been previously used in pregnancy with similar methodology as described in the present protocol [19].

\section{Haematology and biochemical analysis}

Venous blood samples will be extracted in standardized fasting conditions at 8-9 a.m. in the hospital and collected in EDTA vacuum tubes, and tubes for serum. Some samples will be centrifuged at $1750 \mathrm{rpm}$ for $10 \mathrm{~min}$ at $4{ }^{\circ} \mathrm{C}$ in a refrigerated centrifuge (GS-6R Beckman, Fullerton, CA, USA) to separate plasma from formed elements. Subsequently, erythrocytes will be centrifuged in a hypertonic solution to remove membranes and cytosol.
Haematology Erythrocyte count, haematocrit, haemoglobin, platelets, leukocytes and erythrocyte mean corpuscular volume will be quantified by a Coulter counter.

Biochemical parameters Plasma total, high-density lipoprotein and low-density lipoprotein cholesterol, triglycerides, phospholipids, creatine kinase, aspartate aminotransferase, alanine aminotransferase, gammaglutamyl transferase, lactate dehydrogenase, alkaline phosphatase, glucose, urea, insulin, albumin, high sensitivity C-reactive protein, glycosylated haemoglobin, creatinine, total bilirubin, myoglobin, lactate, troponin I, peroxisome proliferator-activated receptor- $\gamma$ coactivator (PGC)-1 $\alpha$, nitric oxide and myeloperoxidase will be assessed with standard methods using an autoanalyzer (Hitachi-Roche p800, F. Hoffmann-La Roche Ltd. Switzerland) or by using commercial kits (spectrophotometry and ELISA).

\section{Oxidative stress}

a) Antioxidant defence

- Plasma measurements: liposoluble antioxidants (vitamin E, retinol, carotene, coenzyme Q10 and coenzyme Q9) through mass spectrometry and the total plasma antioxidant capacity by using commercial kit (spectrophotometry).

- Erythrocyte measurements: liposoluble antioxidants in membrane (vitamin E and coenzyme Q10) through mass spectrometry and antioxidant enzymes activity (catalase, glutathione peroxidase and superoxide dismutase) by spectrophotometry.

\section{b) Oxidative damage}

- Oxidative damage to proteins: measurement of carbonyl proteins by using commercial kit (spectrophotometry).

- Oxidative damage to lipids: measurement of 4hydroxynonenal and isoprostanes in urine and plasma and hydroperoxides in plasma and erythrocyte membrane by using commercial kits (spectrophotometry and ELISA).

- Oxidative damage to DNA: measurement of 8hydroxyguanosine in urine and plasma by commercial kits (ELISA).

\section{Pro- and anti-inflammatory signal}

Some maternal and umbilical cord plasma proinflammatory and anti-inflammatory cytokines (IL-1 $\beta$, IL-2, IL-6, IL-8, IL-10, IFN- $\gamma$ and TNF- $\alpha$, IL-1ra and TNF Srii $\alpha$ ), some adipokines (adiponectin, adipsin, resistin, PAI-active, insulin and leptin) and myokines (irisin) will be measured by the employment of Luminex xMAP technology. 


\section{Bone biomarkers}

Various relevant biomarkers related to bone metabolism (ACTH, DKK-1, FGF-23, Osteocalcin, OPN-Osteopontin, Osteoprotegerin, PTH and SOST) will be measured with Luminex xMAP technology.

\section{Sleep quality}

The Pittsburgh Sleep Quality Index [76] will be used to assess sleep quality and disturbances over a l-month time interval. Nineteen individual items generate seven "component" scores: subjective sleep quality, sleep latency, sleep duration, habitual sleep efficiency, sleep disturbances, use of sleeping medication, and daytime dysfunction. Each component yields a score ranging from 0 to 3, with 3 indicating the greatest dysfunction. The seven component scores are summed to provide a global sleep quality score (range 0 to 21) with higher scores indicating poor sleep quality.

Nevertheless, it has been shown that reported sleep by pregnant women varies in relation to that objectively estimated with accelerometry [77]. Consequently, behaviours related to sleep, such as sleep onset, sleep latency, total sleep time, number and duration of awakenings and sleep efficiency will be objectively calculated by using a triaxial accelerometer (ActiSleep+, Pensacola, Florida, United States). The data analysis of such ActiSleep accelerometry data will be carried out through its specific software (Actilife).

\section{Quality of life, mental health and positive health}

We will use the Short-Form Health Survey 36 [78], for assessing health-related quality of life. It contains 36 items grouped into 8 dimensions: physical functioning, physical role, body pain, general health, vitality, social functioning, emotional role, and mental health. The scores range from 0 to 100 in every dimension, where higher scores indicate better health.

The pregnant antenatal depression will be assessed with the Center for Epidemiological Studies-Depression Scale questionnaire [79], which is validated and widely employed in pregnancy. Postnatal depression levels will be assessed with the Spanish version of the Edinburgh Postnatal Depression Scale [80], which is a 10-items selfreport scale designed as a specific instrument to detect postnatal depression.

Anxiety levels will be assessed with the State Trait Anxiety Index [81]. This questionnaire consists of two scales that assess anxiety state and trait anxiety. The total score ranges from 20 to 80, with higher values indicating higher levels of anxiety.

The "Restless Legs Syndrome" largely determines the quality of life for many pregnant and is related to their levels of depression and sleep quality [82]. The Spanish version of the Detention of Restless Legs Syndrome questionnaire [83] will be employed to assess the presence and severity of this syndrome.

Sexual function is also an important aspect of the quality of life of a person. The Spanish version of the 6item Female Sexual Function Index [84] was used to assess sex function. This instrument is composed of six questions: desire, arousal, lubrication, orgasm, satisfaction and pain. Each question can be scored from 0 to 5 and then summed up to provide a total score.

Finally, "positive health" will be evaluated through the following questionnaires:

1) The Trait Meta-Mood Scale [85] is comprised of 3 subscales to assess participant's beliefs about attending to and value their feelings (emotional attention), feel clear rather than confused about their feelings (emotional clarity) and how well participants regulate their moods and repair negative emotional experiences (emotional repair). Each subscale comprises 8 items. Participants rate their responses using a 5-point Likert type scale, with 1 $=$ "strongly disagree" to $5=$ "strongly agree". The subscales score range from 8 to 40 and higher scores reflect greater attention, clarity, and repair.

2) The Positive and Negative Affect Schedule [86] is a 20-item questionnaire designed to measure positive and negative affect. The questionnaire includes 10 positive and 10 negative emotional states that should be answered on a 5-point Likert scale, from 1 = "very slightly or not at all" to 5 = "extremely". The score ranges from 10 to 50 for both subscales (positive affect and negative affect), and higher scores reflect greater affective well-being.

3) The 10-item Connor-Davidson Resilience Scale [87, 88] assesses resilience to stress, which is a construct refers to a dynamic process of positive adaptation to adverse changes in life circumstances. Each item ranges from $0=$ "not true at all" to $4=$ "true nearly all the time". The total score range from 0 to 40, and higher scores indicate greater resilience.

\section{Statistical analysis}

Since this study aims at determining potential biological effects of exercise, the statistical analysis will be performed on a per-protocol basis, thus, including in the analyses only participants who attended at least $80 \%$ the exercise session and completed both baseline and follow-up evaluations. The treatment effects will be reported as between-group changes on the primary and secondary outcomes and will be assessed with generalized linear models after adjusting for baseline values. All the analyses will be repeated additionally adjusting for age and any other confounder potentially not well balanced at baseline, and the results from both models will 
be reported. The effect size (95\% confidence interval) and statistical significance will be reported for each study outcome. The statistical significance will be set at $\alpha=0.05$. Sensitivity analyses will be conducted using intention-to-treat analyses and baseline observation carried forward imputation.

\section{Discussion}

This paper describes the protocol performed by a multidisciplinary team of experts in PA and exercise, nutrition, gynaecology and physiology that aims to determine the effects of a supervised exercise intervention developed in overweight and grade I obese pregnant on the mother and newborn health, and the influence of lifestyle during pregnancy on relevant, but no explored yet, maternal and foetal outcomes.

The intrauterine environment seems to be involved in programming and foetal offspring exposed to maternal stress, inappropriate diet, physical inactivity, obesity or hyperglycaemia, among others, may be prone to future metabolic alterations and chronic diseases [26, 89-91]. Among these modifiable behaviours, PA is especially relevant during gestation [4-6]. Increasing PA levels or exercising during pregnancy is an efficient tool to tackle cardiometabolic diseases such as GDM, and excessive maternal and neonatal weight gains [5, 19, 92]. However, modern society sometimes treats pregnancy as a disease. For example, it qualifies as vulnerable women and are excluded from most studies [93]. Furthermore, is noticeably that most of the interventions focus on improving pregnant health involve mere advices or counselling given face to face, or by phone (or both). Just few trials have investigated well-controlled individually tailored exercise interventions. Consequently, further trials, with objective outcome measures, are needed. Moreover, the influence of physical fitness on important maternal health markers during gestation, such as quality of life, positive health, inflammatory profile, bone health, or oxidative stress is unknown.

Exercise during pregnancy is safe [21, 22] and sideeffects are minimal $[53,54]$ if pregnant adequate the intensity of such exercise to warrant foetal wellbeing [94]. However, the exercise intensity suggested by the ACOG in 2002 [53] and by Davenport et al. [95] will not be adopted in the present RCT. This is firstly, because the minimum work intensity is stated at $101 \mathrm{bpm}$ for all women (which constitute a low heart rate), and secondly, because they make an absolute estimation (independently of the woman's age, basal heart rate, or physical fitness status). Thereby, in agreement with Zavorsky \& Longo [55], we advocate for modulating the intensity based on the \% HRR, and the RPE.

Not less important for an optimal pregnancy is a healthy psychological status $[31,32,96]$. In that sense, exercise represent an excellent opportunity to improve quality of life [33] and reduce cortisol levels, which may in turn have a positively influence on the foetus and child [31, 32]. Besides, it is unknown whether exercise could positively influence the characteristic worse sleep quality during gestation [48, 49], which can alter the pregnant metabolic status [41-43] and gestational outcomes [44-46] and negatively affect the pregnant quality of life [37-40] and the foetal development [47].

There is a clear and compelling rationale for increased pregnancy research in order to address the therapeutic needs of pregnant women [93]. Additionally, there is accumulating evidence that pregnancy provides a unique window into understanding fundamental mechanisms underlying observed links between a pregnant woman's health and her later health and the health of her children [93]. The information obtained from this RCT will therefore be of clinical and public health interest and will suggest future research. Further, the exercise intervention designed is novel and non-expensive, and can be easily transferred to other similar contexts. Consequently, the findings of the GESTAFIT Project will help the Health Systems to identify strategies for primary prevention and health promotion among overweight and obese pregnant.

\section{Abbreviations \\ GDM: Gestational diabetes mellitus; HRR: Heart rate reserve; PA: Physical activity; RCT: Randomised controlled trial; RPE: Rate of perceived exertion; $\mathrm{SD}$ : Standard deviation; $\mathrm{VO}_{2 \mathrm{max}}$ : Maximal oxygen uptake \\ Funding \\ This project is part of VAA fellowship and was approved by the Andalucía Talent-Hub Program, launched by the Andalusian Knowledge Agency, co-funded by the European Union's Seventh Framework Program, Marie Skłodowska-Curie actions (COFUND-Grant Agreement $n^{\circ} 291780$ ) and the Ministry of Economy, Innovation, Science and Employment of the Junta de Andalucía. ICR (BES-2011- 047133) is supported by the Spanish Ministry of Education. FEL (BES-2014- 067612) is supported by the Spanish Ministry of Economy and Competitiveness.}

Availability of data and materials

Not applicable.

\section{Authors' contributions}

VAA, MNVP, OO, CPV, ASM and JJOH conceived the study and participated in its design. VAA drafted the manuscript. PRC, MDF, JRR and FEL participated in the study design and critically revised the manuscript. LRG, ICAG, VAA, $M B C, I C R$ and PA participated in the study design, critically revised the manuscript and designed and supervised the exercise intervention. All authors read and approved the final manuscript.

\section{Competing interests}

The authors declare that they have no competing interests.

\section{Consent for publication}

Not applicable. The present manuscript does not contain any individual person's data in any form.

\section{Ethics approval and consent to participate}

This study was approved by the Clinical Research Ethics Committee of Granada, Government of Andalusia, Spain (code: GESFIT-0448-N-15). The funding source was not commercial, it belong to a Fellowship from the first author of this manuscript, and had undergone peer review process. Participants will have to provide a written informed consent before taking part in the study, which will be conducted in accordance with the CONSORT (Consolidated Standards of Reporting Trials) statement [50]. 


\section{Author details}

'Department of Physiology, Faculty of Pharmacy and Institute of Nutrition and Food Technology, University of Granada, Granada, Spain. ${ }^{2}$ Department of Public and Occupational Health, and EMGO+ Institute for Health and Care Research, VU University medical center, Amsterdam, The Netherlands. ${ }^{3}$ Department of Obstetrics and Gynecology, University of Granada, Granada, Spain. ${ }^{4}$ Obstetrics and Gynecology Service, University Hospital Complex, Granada, Spain. ${ }^{5}$ Department of Education, Faculty of Education Sciences, University of Almería, Almería, Spain. ${ }^{6}$ Department of Physical Education and Sports, Faculty of Sport Sciences, University of Granada, Granada, Spain. ${ }^{7}$ Department of Clinical and Health Psychology, Faculty of Social and Behavioral Sciences, Utrecht University, Utrecht, The Netherlands. ${ }^{8}$ Institute of Sport Science, University of Graz, Graz, Austria.

\section{Received: 10 November 2015 Accepted: 22 September 2016} Published online: 29 September 2016

\section{References}

1. Barouki R, Gluckman PD, Grandjean P, Hanson M, Heindel JJ. Developmental origins of non-communicable disease: implications for research and public health. Environ Health. 2012;11:42.

2. Hanson MA, Gluckman PD. Developmental origins of health and diseaseglobal public health implications. Best pract res Clin obstetrics gynaecol. 2015:29(1):24-31.

3. Hure AJ, Collins CE, Giles WB, Wright IM, Smith R. Protocol for the Women And Their Children's Health (WATCH) study: a cohort of pregnancy and beyond. J epidemiol. 2012;22(3):267-75.

4. Loprinzi PD, Fitzgerald EM, Woekel E, Cardinal BJ. Association of physical activity and sedentary behavior with biological markers among U.S. pregnant women. J women's health (2002). 2013;22(11):953-8.

5. de Groot CJ, van Poppel MN, van Poppel MN, Ruchat SM, Mottola MF. Physical activity and gestational diabetes mellitus. J pregnancy. 2014:60:104-12

6. Mudd LM, Evenson KR. Review of impacts of physical activity on maternal metabolic health during pregnancy. Curr diabetes reports. 2015;15(2):572.

7. Melzer K, Schutz Y, Boulvain M, Kayser B. Physical activity and pregnancy: cardiovascular adaptations, recommendations and pregnancy outcomes. Sports med (Auckland, NZ). 2010;40(6):493-507.

8. Dempsey JC, Butler CL, Williams MA. No need for a pregnant pause: physical activity may reduce the occurrence of gestational diabetes mellitus and preeclampsia. Exerc Sport Sci Rev. 2005;33(3):141-9.

9. Boghossian NS, Yeung E, Albert PS, Mendola P, Laughon SK, Hinkle SN, Zhang C. Changes in diabetes status between pregnancies and impact on subsequent newborn outcomes. Am J Obstet Gynecol. 2014;210(5):431. e431-414.

10. Cnattingius S, Villamor E, Johansson S, Edstedt Bonamy AK, Persson M, Wikstrom AK, Granath F. Maternal obesity and risk of preterm delivery. Jama. 2013;309(22):2362-70.

11. Persson M, Johansson S, Villamor E, Cnattingius S. Maternal overweight and obesity and risks of severe birth-asphyxia-related complications in term infants: a population-based cohort study in Sweden. PLoS Med. 2014;11(5):e1001648.

12. Lee KK, Raja EA, Lee AJ, Bhattacharya S, Bhattacharya S, Norman JE, Reynolds RM. Maternal Obesity During Pregnancy Associates With Premature Mortality and Major Cardiovascular Events in Later Life. Hypertension. 2015;66(5):938-44.

13. Pacce S, Saure C, Mazza CS, Garcia S, Tomzig RG, Lopez AP, Ribarola L, Krochick GA. Impact of maternal nutritional status before and during pregnancy on neonatal body composition: A cross-sectional study. Diabetes metabolic syndr. 2015;10:S7-S12.

14. Carty D, Akehurst C, Savage R, Sungatullina L, Robinson S, McBride M, McClure J, Freeman D, Delles C. Differential gene expression in obese pregnancy. Pregnancy hypertension. 2014;4(3):232-3.

15. Awan S, Bibi S, Makhdoom A, Farooq S, Sm T, Qazi RA. Adverse fetomaternal outcome among pregnant overweight women. Pakistan j med sci. 2015;31(2):383-7

16. Johansson S, Villamor E, Altman M, Bonamy AK, Granath F, Cnattingius S. Maternal overweight and obesity in early pregnancy and risk of infant mortality: a population based cohort study in Sweden. BMJ. 2014;349:96572.

17. Nascimento SL, Surita FG, Godoy AC, Kasawara KT, Morais SS. Physical Activity Patterns and Factors Related to Exercise during Pregnancy: A Cross Sectional Study. PLoS One. 2015;10(6):e0128953.
18. Clarke PE, Gross H. Women's behaviour, beliefs and information sources about physical exercise in pregnancy. Midwifery. 2004;20(2):133-41.

19. Ruifrok AE, Althuizen E, Oostdam N, van Mechelen W, Mol BW. The relationship of objectively measured physical activity and sedentary behaviour with gestational weight gain and birth weight. J Pregnancy. 2014;2014:567379. doi:10.1155/2014/567379.

20. Ruiz JR, Perales M, Pelaez M, Lopez C, Lucia A, Barakat R. Supervised exercise-based intervention to prevent excessive gestational weight gain: a randomized controlled trial. Mayo Clin Proc. 2013;88(12):1388-97.

21. Petrov Fieril K, Glantz A, Fagevik Olsen M. The efficacy of moderate-tovigorous resistance exercise during pregnancy: a randomized controlled trial. Acta Obstet Gynecol Scand. 2015;94(1):35-42.

22. O'Connor PJ, Poudevigne MS, Cress ME, Motl RW, Clapp 3rd JF. Safety and efficacy of supervised strength training adopted in pregnancy. J Phys Act Health. 2011:8(3):309-20.

23. Benjamin DR, van de Water AT, Peiris CL. Effects of exercise on diastasis of the rectus abdominis muscle in the antenatal and postnatal periods: a systematic review. Physiotherapy. 2014;100(1):1-8.

24. Hall DC, Kaufmann DA. Effects of aerobic and strength conditioning on pregnancy outcomes. Am J Obstet Gynecol. 1987;157(5):1199-203.

25. Brankston GN, Mitchell BF, Ryan EA, Okun NB. Resistance exercise decreases the need for insulin in overweight women with gestational diabetes mellitus. Am J Obstet Gynecol. 2004;190(1):188-93.

26. Hazeldean D. Being fit in pregnancy. pract midwife. 2014;17(2):11-2. 14.

27. Bisson M, Almeras N, Plaisance J, Rheaume C, Bujold E, Tremblay A, Marc L. Maternal fitness at the onset of the second trimester of pregnancy: correlates and relationship with infant birth weight. Pediatr obesity. 2013:8(6):464-74.

28. Clapp 3rd JF. Morphometric and neurodevelopmental outcome at age five years of the offspring of women who continued to exercise regularly throughout pregnancy. J Pediatr. 1996;129(6):856-63

29. Park JW, Kim MH, Eo SJ, Lee EH, Kang JS, Chang HK, Leem YH. Maternal exercise during pregnancy affects mitochondrial enzymatic activity and biogenesis in offspring brain. Int j neurosci. 2013;123(4):253-64.

30. May LE, Scholtz SA, Suminski R, Gustafson KM. Aerobic exercise during pregnancy influences infant heart rate variability at one month of age. Early Hum Dev. 2014;90(1):33-8.

31. Keshavarzi F, Farnia V, Yazdchi K, Najafi F, Brand S, Bajoghli H, Nankali A, Salmanzadeh $\mathrm{H}$. Effect of maternal anxiety on maternal serum and fetal cord blood cortisol. Asia-Pacific psychiatry. 2014;6(4):435-9.

32. Tapia Collados C, Gil Guillen V, Orozco Beltran D. [Influence of maternal anxiety on the frequency of paediatric primary care visits. Atencion primaria/Sociedad Espanola de Medicina de Familia y Comunitaria. 2005:36(2):64-8.

33. Kolu P, Raitanen J, Luoto R. Physical activity and health-related quality of life during pregnancy: a secondary analysis of a cluster-randomised trial. Matern Child Health J. 2014;18(9):2098-105.

34. Gaston A, Prapavessis H. Tired, moody and pregnant? Exercise may be the answer. Psychol Health. 2013;28(12):1353-69.

35. Poudevigne MS, O'Connor PJ. A review of physical activity patterns in pregnant women and their relationship to psychological health. Sports med (Auckland, NZ). 2006;36(1):19-38.

36. Shivakumar G, Brandon AR, Snell PG, Santiago-Munoz P, Johnson NL, Trivedi $\mathrm{MH}$, Freeman MP. Antenatal depression: a rationale for studying exercise. Depression anxiety. 2011;28(3):234-42.

37. Rezaei E, Moghadam ZB, Saraylu K. Quality of life in pregnant women with sleep disorder. J family reproduct health. 2013;7(2):87-93.

38. Wu M, Li X, Feng B, Wu H, Qiu C, Zhang W. Correlation between sleep quality of third-trimester pregnancy and postpartum depression. Med Sci Monit. 2014:20:2740-5

39. Okun ML, Kline CE, Roberts JM, Wettlaufer B, Glover K, Hall M. Prevalence of sleep deficiency in early gestation and its associations with stress and depressive symptoms. J women's health (2002). 2013;22(12):1028-37.

40. Pires GN, Andersen ML, Giovenardi M, Tufik S. Sleep impairment during pregnancy: possible implications on mother-infant relationship. Med Hypotheses. 2010;75(6):578-82.

41. Hayase M, Shimada M, Seki H. Sleep quality and stress in women with pregnancy-induced hypertension and gestational diabetes mellitus. Women birth. 2014:27(3):190-5.

42. Izci Balserak B, Jackson N, Ratcliffe SA, Pack Al, Pien GW. Sleep-disordered breathing and daytime napping are associated with maternal hyperglycemia. Sleep breathing. 2013;17(3):1093-102. 
43. Okun ML, Luther JF, Wisniewski SR, Wisner KL. Disturbed sleep and inflammatory cytokines in depressed and nondepressed pregnant women: an exploratory analysis of pregnancy outcomes. Psychosom Med. 2013;75(7):670-81.

44. Okun ML, Schetter CD, Glynn LM. Poor sleep quality is associated with preterm birth. Sleep. 2011;34(11):1493-8.

45. Owusu JT, Anderson FJ, Coleman J, Oppong S, Seffah JD, Aikins A, O'Brien LM. Association of maternal sleep practices with pre-eclampsia, low birth weight, and stillbirth among Ghanaian women. Int j gynaecol obstetrics. 2013;121(3):261-5.

46. Ko YL, Lin PC, Chen SC. Stress, sleep quality and unplanned Caesarean section in pregnant women. Int j nurs pract. Int J Nurs Pract. 2015;21(5):454-61. doi:10.1111/ijn.12267.

47. Hung HM, Ko SH, Chen $\mathrm{CH}$. The association between prenatal sleep quality and obstetric outcome. j nurs res. 2014;22(3):147-54.

48. Madaeva IM, Kolesnikova LI, Protopopova NV, Sakh'ianova NL, Berdina ON. [Features of the sleep pattern during pregnancy]. Vestn Ross Akad Med Nauk. 2014;1-2:93-7.

49. Lara-Carrasco J, Simard V, Saint-Onge K, Lamoureux-Tremblay V, Nielsen T. Disturbed dreaming during the third trimester of pregnancy. Sleep Med. 2014;15(6):694-700.

50. Schulz KF, Altman DG, Moher D. CONSORT 2010 statement: updated guidelines for reporting parallel group randomized trials. Obstet Gynecol. 2010;115(5):1063-70.

51. Kanagasabai T, Thakkar NA, Kuk JL, Churilla JR, Ardern Cl. Differences in physical activity domains, guideline adherence, and weight history between metabolically healthy and metabolically abnormal obese adults: a crosssectional study. int j behav nutr phys act. 2015;12:64.

52. Kalisiak B, Spitznagle T. What effect does an exercise program for healthy pregnant women have on the mother, fetus, and child? PM R: j injury, function, rehabilitation. 2009;1(3):261-6.

53. ACOG committee opinion. Exercise during pregnancy and the postpartum period. Number 267, January 2002. American College of Obstetricians and Gynecologist. Int J Gynaecol Obstet. 2002;77(1):79-81.

54. Evenson KR, Barakat R, Brown WJ, Dargent-Molina P, Haruna M, Mikkelsen EM, Mottola MF, Owe KM, Rousham EK, Yeo S. Guidelines for Physical Activity during Pregnancy: Comparisons From Around the World. Am J Lifestyle Med. 2014;8(2):102-21.

55. Zavorsky GS, Longo LD. Exercise guidelines in pregnancy: new perspectives. Sports med(Auckland, NZ). 2011;41(5):345-60.

56. Barakat R, Pelaez M, Lopez C, Lucia A, Ruiz JR. Exercise during pregnancy and gestational diabetes-related adverse effects: a randomised controlled trial. Br J Sports Med. 2013;47(10):630-6.

57. White E, Pivarnik J, Pfeiffer K. Resistance training during pregnancy and perinatal outcomes. J Phys Act Health. 2014;11(6):1141-8.

58. Pelaez M, Gonzalez-Cerron S, Montejo R, Barakat R. Pelvic floor muscle training included in a pregnancy exercise program is effective in primary prevention of urinary incontinence: a randomized controlled trial. Neurourol Urodyn. 2014;33(1):67-71.

59. Medicine ACOS. ACSM's Guidelines for Exercise Testing and Prescription. ninth editionth ed. Philadelphia: Lippincott Williams and Wilkins; 2013.

60. Bozkurt M, Yumru AE, Sahin L. Pelvic floor dysfunction, and effects of pregnancy and mode of delivery on pelvic floor. Taiwanese j obstetr gynecol. 2014;53(4):452-8.

61. Borg GA. Psychophysical bases of perceived exertion. Med Sci Sports Exerc 1982;14(5):377-81

62. Okamoto T, Masuhara M, Ikuta K. Acute effects of self-myofascial release using a foam roller on arterial function. J strength condition res. 2014;28(1):69-73.

63. Ana Carbonell Baeza VAAGM, Jonatan Ruz Ruiz, Francisco B. Ortega Porcel, Manuel Delgado Fernández. Guía de promoción para la actividad física. Junta de Andalucía, Consejería de Salud Depósito Legal: SE-8656-2010 2012.

64. Hui AL, Ludwig S, Gardiner P, Sevenhuysen G, Dean HJ, Sellers E, McGavock J, Morris M, Shen GX, Jiang D. Effects of lifestyle intervention on dietary intake, physical activity level, and gestational weight gain in pregnant women with different pre-pregnancy Body Mass Index in a randomized control trial. BMC pregnancy childbirth. 2014;14(1):331.

65. de Onis M, Habicht JP. Anthropometric reference data for international use: recommendations from a World Health Organization Expert Committee. Am J Clin Nutr. 1996;64(4):650-8. http://ajcn.nutrition.org/content/64/4/650.long.

66. Perin F, Pittet JC, Schnebert S, Perrier P, Tranquart F, Beau P. Ultrasonic assessment of variations in thickness of subcutaneous fat during the normal menstrual cycle. European j ultrasound. 2000;11(1):7-14.
67. Panagiotakos DB, Pitsavos C, Stefanadis C. Dietary patterns: a Mediterranean diet score and its relation to clinical and biological markers of cardiovascular disease risk. Nutr Metab Cardiovasc Dis. 2006;16(8):559-68.

68. Mariscal-Arcas M, Rivas A, Monteagudo C, Granada A, Cerrillo I, Olea-Serrano F. Proposal of a Mediterranean diet index for pregnant women. Br J Nutr. 2009;102(5):744-9.

69. Mataix J L, Martinez de Victoria E. et al. Valoración del estado nutricional de la Comunidad Autónoma de Andalucía; 2000

70. Astrand PO, Ryhming I. A nomogram for calculation of aerobic capacity (physical fitness) from pulse rate during sub-maximal work. J Appl Physiol. 1954;7(2):218-21.

71. Rikli RE, Jones J. Development and validation of a functional fitness test for community residing older adults. J Aging Phys Act. 1999;7:129-61.

72. Ruiz-Ruiz J, Mesa JL, Gutierrez A, Castillo MJ. Hand size influences optimal grip span in women but not in men. J Hand Surg [Am]. 2002;27(5):897-901.

73. Ortega FB, Ruiz JR, Espana-Romero V, Vicente-Rodriguez G, Martinez-Gomez D, Manios Y, Beghin L, Molnar D, Widhalm K, Moreno LA, et al. The International Fitness Scale (IFIS): usefulness of self-reported fitness in youth. Int J Epidemiol. 2011;40(3):701-11.

74. Payares K, Lugo LH, Morales V, Londono A. Validation in Colombia of the Oswestry disability questionnaire in patients with low back pain. Spine. 2011;36(26):E1730-5

75. Bijur PE, Silver W, Gallagher EJ. Reliability of the visual analog scale for measurement of acute pain. Acad Emerg Med. 2001;8(12):1153-7.

76. Buysse DJ, Reynolds 3rd CF, Monk TH, Berman SR, Kupfer DJ. The Pittsburgh Sleep Quality Index: a new instrument for psychiatric practice and research. Psychiatry Res. 1989;28(2):193-213.

77. Herring SJ, Foster GD, Pien GW, Massa K, Nelson DB, Gehrman PR, Davey A. Do pregnant women accurately report sleep time? A comparison between self-reported and objective measures of sleep duration in pregnancy among a sample of urban mothers. Sleep breathing. 2013;17(4):1323-7.

78. Alonso J, Prieto L, Anto JM. [The Spanish version of the SF-36 Health Survey (the SF-36 health questionnaire): an instrument for measuring clinical results]. Med Clin (Barc). 1995;104(20):771-6.

79. Ruiz-Grosso P, Loret de Mola C, Vega-Dienstmaier JM, Arevalo JM, Chavez K, Vilela A, Lazo M, Huapaya J. Validation of the Spanish Center for Epidemiological Studies Depression and Zung Self-Rating Depression Scales: a comparative validation study. PLoS One. 2012;7(10):e45413.

80. Garcia-Esteve L, Ascaso C, Ojuel J, Navarro P. Validation of the Edinburgh Postnatal Depression Scale (EPDS) in Spanish mothers. J Affect Disord. 2003;75(1):71-6.

81. Spielberger CD, Gorsuch RL, RE. L (eds.). STAl: Manual del Cuestionario de Ansiedad Estado-Rasgo. Madrid: TEA Ediciones; 2002.

82. Wesstrom J, Skalkidou A, Manconi M, Fulda S, Sundstrom-Poromaa I. Prepregnancy restless legs syndrome (Willis-Ekbom Disease) is associated with perinatal depression. J Clin Sleep Med. 2014;10(5):527-33.

83. Perez-Romero T, Comas-Fuentes A, Deban-Fernandez M, Gonzalez-Nuevo Quinones JP, Maujo-Fernandez J. [Prevalence of restless legs syndrome at the office in primary health care]. Rev Neurol. 2007:44(11):647-51.

84. Isidori AM, Pozza C, Esposito K, Giugliano D, Morano S, Vignozzi L, Corona G, Lenzi A, Jannini EA. Development and validation of a 6 -item version of the female sexual function index (FSFI) as a diagnostic tool for female sexual dysfunction. J Sex Med. 2010;7(3):1139-46.

85. Fernandez-Berrocal P, Extremera N, Ramos N. Validity and reliability of the Spanish modified version of the Trait Meta-Mood Scale. Psychol Rep. 2004; 94(3 Pt 1):751-5

86. Watson D, Clark LA, Tellegen A. Development and validation of brief measures of positive and negative affect: the PANAS scales. J Pers Soc Psychol. 1988;54(6):1063-70.

87. Campbell-Sills L, Stein MB. Psychometric analysis and refinement of the Connor-davidson Resilience Scale (CD-RISC): Validation of a 10-item measure of resilience. J Trauma Stress. 2007;20(6):1019-28.

88. Notario-Pacheco B, Solera-Martinez M, Serrano-Parra MD, BartolomeGutierrez R, Garcia-Campayo J, Martinez-Vizcaino V. Reliability and validity of the Spanish version of the 10-item Connor-Davidson Resilience Scale (10item (D-RISC) in young adults. Health qual life outcomes. 2011;9:63.

89. Junien C. Early determinants of health and disease: epigenetics and environment. Bull Acad Natl Med. 2011;195(3):511-26. discussion 526-517.

90. Gaudet L, Wen SW, Walker M. The combined effect of maternal obesity and fetal macrosomia on pregnancy outcomes. J Obstet Gynaecol Can. 2014;36(9):776-84 
91. Sebert SP, Hyatt MA, Chan LL, Yiallourides M, Fainberg HP, Patel N, Sharkey D, Stephenson T, Rhind SM, Bell RC, et al. Influence of prenatal nutrition and obesity on tissue specific fat mass and obesity-associated (FTO) gene expression. Repro (Cambridge, England). 2010;139(1):265-74.

92. Russo LM, Nobles C, Ertel KA, Chasan-Taber L, Whitcomb BW. Physical Activity Interventions in Pregnancy and Risk of Gestational Diabetes Mellitus: A Systematic Review and Meta-analysis. Obstet Gynecol. 2015;125(3):576-82.

93. Blehar MC, Spong C, Grady C, Goldkind SF, Sahin L, Clayton JA. Enrolling pregnant women: issues in clinical research. Women's health issues. 2013;23(1):e39-45.

94. Salvesen $K \AA ̊$, Hem E, Sundgot-Borgen J. Fetal wellbeing may be compromised during strenuous exercise among pregnant elite athletes. Br J Sports Med. 2012;46(4):279-83.

95. Davenport MH, Charlesworth S, Vanderspank D, Sopper MM, Mottola MF. Development and validation of exercise target heart rate zones for overweight and obese pregnant women. Appl physiol nutr metabol. 2008;33(5):984-9.

96. Rallis S, Skouteris H, McCabe M, Milgrom J. A prospective examination of depression, anxiety and stress throughout pregnancy. Women birth. 2014;27(4):e36-42

Submit your next manuscript to BioMed Central and we will help you at every step:

- We accept pre-submission inquiries

- Our selector tool helps you to find the most relevant journal

- We provide round the clock customer support

- Convenient online submission

- Thorough peer review

- Inclusion in PubMed and all major indexing services

- Maximum visibility for your research

Submit your manuscript at www.biomedcentral.com/submit
Biomed Central 